\title{
Fast multipole boundary element method for the simulation of acoustic-structure interaction
}

\author{
L. Gaul \& M. Fischer \\ Institute of Applied and Experimental Mechanics, \\ University of Stuttgart, Germany
}

\begin{abstract}
A fast multilevel multipole algorithm (FMM) is derived for the Helmholtz equation and adopted to the symmetric Galerkin boundary element method (BEM) for acoustics. The FMM allows to evaluate a matrix-vector product of the BEM with a computational cost of $\mathcal{O}\left(N \log ^{2} N\right)$, thus leading to a significant reduction of computation time and memory requirements compared to standard BEM formulations. This allows the simulation of large scale acoustic models. A coupling algorithm based on Lagrange multipliers is proposed for the simulation of structure-acoustic field interaction. Finite plate elements are coupled to a Galerkin boundary element formulation of the acoustic domain. The interface pressure is interpolated as a Lagrange multiplier, thus, allowing the coupling of non-matching grids. The resulting saddle point problem is solved by an approximate Uzawa-type scheme in which the matrix-vector products of the boundary element operators are evaluated efficiently by the fast multipole boundary element method. The algorithm is demonstrated on the example of a cavity backed elastic panel.

Keywords: fast multipole Galerkin BEM, acoustic-structure interaction, mortar BEM-FEM coupling.
\end{abstract}

\section{Introduction}

The application of the fast multipole BEM for the simulation of acoustic fieldstructure interaction problems poses new demands on the solver for the coupled systems: direct approaches are not applicable and iterative schemes suffer from illconditioning. An approximate Uzawa-type algorithm is proposed for the solution of saddle point systems arising from mortar coupling. The efficiency of the nested 
iterative solver is improved by a relaxation scheme and preconditioning of the subsystems.

\section{Acoustic-structure interaction}

A structure fully submerged in an acoustic fluid as displayed in Fig. 1 is modeled as a thin Kirchhoff plate on the interaction boundary $\Gamma^{\text {int }}$. The out-of-plane displacement is denoted by $w$, the loading $f=f^{\mathrm{f}}+f^{\mathrm{e}}$ consists of surface forces due to the acoustic field $f^{\mathrm{f}}$ and externally applied forces $f^{\mathrm{e}}$. The timeharmonic pressure $p$ in the acoustic field $\Omega_{\mathrm{f}}$ is governed by the Helmholtz equation $\triangle p+\kappa^{2} p=0$ with the circular wavenumber $\kappa=\omega / c_{\mathrm{f}}$. The acoustic flux on the boundary is defined as $q=\partial p / \partial \vec{n}_{\mathrm{f}}$. For simplicity of presentation, the boundary $\partial \Omega_{\mathrm{f}}=\Gamma=\Gamma^{\mathrm{int}} \cup \Gamma^{\mathrm{N}}$ is composed of acoustic-structure interface and Neumann boundary. Dirichlet boundary conditions or computations on exterior domains can be implemented without difficulties. On the acoustic-structure interface $\Gamma^{\text {int }}$ the coupling conditions enforce equilibrium $p=f^{\mathrm{f}}$ and continuity $q=-\rho_{\mathrm{f}} \omega^{2} w$.

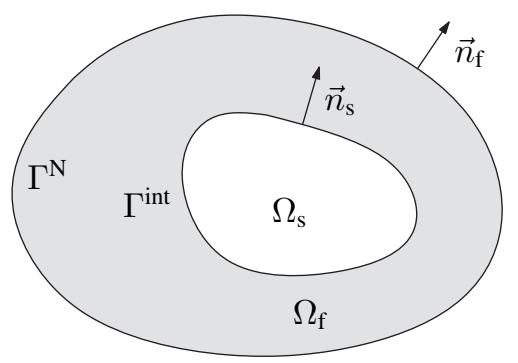

Figure 1: Structure and acoustic domain.

\section{Mortar coupling}

A mortar algorithm is employed for the BEM-FEM coupling that allows a nonconforming discretization of the sub-domains. The BEM mesh is chosen as mortar side and the interface pressure is interpolated as Lagrange multiplier $\lambda=p=f^{0}$.

The FEM for the Kirchhoff plate is derived from the variational formulation

$$
a\left(\nabla w, \nabla v^{w}\right)-\int_{\Gamma^{\mathrm{int}}} v^{w} \lambda \mathrm{d} s_{x}=\int_{\Gamma^{\text {int }}} v^{w} f^{\mathrm{e}} \mathrm{d} s_{x} .
$$

The BEM for the acoustic fluid is derived from the boundary integral equation

$$
p(x)=\frac{1}{2} p(x)+\underbrace{\int_{\Gamma} P^{*}(x, y) q(y) \mathrm{d} s_{y}}_{(V q)(x)}-\underbrace{\int_{\Gamma} \frac{\partial P^{*}(x, y)}{\partial n_{y}} p(y) \mathrm{d} s_{y}}_{(K p)(x)}, \quad x \in \Gamma,
$$


and the hyper-singular boundary integral equation

$$
q(x)=\frac{1}{2} q(x)+\underbrace{\int_{\Gamma} \frac{\partial P^{*}(x, y)}{\partial n_{x}} q(y) \mathrm{d} s_{y}}_{\left(K^{\prime} q\right)(x)}-\underbrace{\int_{\Gamma} \frac{\partial^{2} P^{*}(x, y)}{\partial n_{x} \partial n_{y}} p(y) \mathrm{d} s_{y}}_{-(D p)(x)}, \quad x \in \Gamma .
$$

The single layer potential $(V q)(x)$, double layer potential $(K p)(x)$, adjoint double layer potential $\left(K^{\prime} q\right)(x)$ and the hyper-singular operator $(D p)(x)$ are the wellknown boundary integral operators with the fundamental solution $P^{*}(x, y)=$ $\mathrm{e}^{\mathrm{i} \kappa|x-y|} /(4 \pi|x-y|)$ defining the integration kernels.

The pressure and flux fields on the boundary are decomposed as $p=p^{\text {int }}+\tilde{p}$ and $q=q^{\text {int }}+\bar{q}$, where $\bar{q}$ are the prescribed Neumann boundary conditions and $\tilde{p}=\bar{q}=0$ on $\Gamma^{\text {int }}$.

Using equation (2) tested with $v^{q}$ on $\Gamma^{\text {int }}$ and equation (3) tested with $v^{p}$ on the entire boundary $\Gamma$, one obtains the system

$$
\begin{gathered}
\int_{\Gamma^{\mathrm{int}}} v^{q}\left(V q^{\mathrm{int}}\right)(x) \mathrm{d} s_{x}-\int_{\Gamma_{\mathrm{int}}} v^{q}(K \tilde{p})(x) \mathrm{d} s_{x}+\int_{\Gamma_{\mathrm{int}}} v^{q}\left[-\frac{1}{2} p^{\mathrm{int}}(x)-\right. \\
\left.\left(K p^{\mathrm{int}}\right)(x)\right] \mathrm{d} s_{x}+\int_{\Gamma^{\mathrm{int}}}\left[p^{\mathrm{int}}(x)-\lambda(x)\right] \mathrm{d} s_{x}=-\int_{\Gamma^{\mathrm{int}}}^{q}(V \bar{q})(x) \mathrm{d} s_{x}, \\
\int_{\Gamma} v^{p}\left(D p^{\mathrm{int}}\right)(x) \mathrm{d} s_{x}+\int_{\Gamma} v^{p}(D \tilde{p})(x) \mathrm{d} s_{x}+\int_{\Gamma} v^{p}\left[-\frac{1}{2} q^{\mathrm{int}}(x)+\right. \\
\left.\left(K^{\prime} q^{\mathrm{int}}\right)(x)\right] \mathrm{d} s_{x}=\int_{\Gamma} v^{p}\left[\frac{1}{2} \bar{q}(x)-\left(K^{\prime} \bar{q}\right)(x)\right] \mathrm{d} s_{x} .
\end{gathered}
$$

The term $p^{\text {int }}(x)-\lambda(x)$ in equation (4) was introduced to enforce equilibrium on the interface. Continuity is enforced by

$$
\int_{\Gamma^{\mathrm{int}}} v^{\lambda}\left(\rho_{\mathrm{f}} \omega^{2} w+q^{\mathrm{int}}\right) \mathrm{d} s_{x}=0 .
$$


Discretizing plate equation (1), fluid equations (4), (5) and continuity equation (6), one obtains the system of equations

$$
\begin{array}{r}
\left(\begin{array}{ccccc}
\rho_{\mathrm{f}} \omega^{2} \mathbf{A} & \mathbf{0} & \mathbf{0} & \mathbf{0} & -\mathbf{C}_{\mathrm{FEM}} \\
\mathbf{0} & \mathbf{V} & \frac{\mathbf{1}}{\mathbf{2}}-\mathbf{K}_{\mathrm{int}, \text { int }} & -\mathbf{K}_{\mathrm{int}, \mathrm{N}} & -\mathbf{C}_{\mathrm{BEM}} \\
\mathbf{0} & -\frac{\mathbf{1}}{\mathbf{2}}+\mathbf{K}_{\mathrm{int}, \mathrm{int}}^{\mathrm{T}} & \mathbf{D}_{\text {int,int }} & \mathbf{D}_{\mathrm{int}, \mathrm{N}} & \mathbf{0} \\
\mathbf{0} & \mathbf{K}_{\mathrm{N}, \mathrm{int}} & \mathbf{D}_{\mathrm{N}, \text { int }} & \mathbf{D}_{\mathrm{N}, \mathrm{N}} & \mathbf{0} \\
\mathbf{C}_{\mathrm{FEM}}^{\mathrm{T}} & \mathbf{C}_{\mathrm{BEM}}^{\mathrm{T}} & \mathbf{0} & \mathbf{0} & \mathbf{0}
\end{array}\right)\left(\begin{array}{c}
\mathbf{w} \\
\mathbf{q}^{\mathrm{int}} \\
\mathbf{p}^{\mathrm{int}} \\
\tilde{\mathbf{p}} \\
\lambda
\end{array}\right) \\
=\left(\begin{array}{c}
\rho_{\mathrm{f}} \omega^{2} \int_{\Gamma^{\mathrm{int}}} \varphi^{w} f^{\mathrm{e}} \mathrm{d} s_{x} \\
-\int_{\Gamma^{\mathrm{int}}} \varphi^{q}(V \bar{q})(x) \mathrm{d} s_{x} \\
\int_{\Gamma} \varphi^{p}\left[\frac{1}{2} \bar{q}(x)-\left(K^{\prime} \bar{q}\right)(x)\right] \mathrm{d} s_{x} \\
\mathbf{0}
\end{array}\right) .
\end{array}
$$

\section{Iterative solution of saddle point problem}

For the solution of the system (7) an approximate Uzawa type algorithm is employed, that requires the evaluation of matrix-vector products of the discretized boundary integral operators. Using a standard BEM formulation, the computing time and memory requirements are of order $\mathcal{O}\left(N^{2}\right)$ and the method is thus not feasible for large scale simulations. Using the fast multipole BEM, the numerical cost can be reduced to $\mathcal{O}\left(N \log ^{2} N\right)$. For a description of the multipole algorithm and the fast realization of the boundary integral operators it is referred to [1].

In the Uzawa algorithm, the system equation (7) is solved for $\boldsymbol{\lambda}$ and GMRES iterations are applied on the reduced equation

$$
\underbrace{\left(\begin{array}{ll}
\mathbf{C}_{\mathrm{FEM}}^{\mathrm{T}} & \mathbf{C}_{\mathrm{BEM}}^{\mathrm{T}}
\end{array}\right)\left(\begin{array}{cc}
\left(\rho_{\mathrm{f}} \omega^{2} \mathbf{A}\right)^{-1} & \mathbf{0} \\
\mathbf{0} & \mathbf{B}^{-1}
\end{array}\right)\left(\begin{array}{c}
\mathbf{C}_{\mathrm{FEM}} \\
\mathbf{C}_{\mathrm{BEM}}
\end{array}\right)}_{\mathbf{S}} \boldsymbol{\lambda}=\underbrace{\left(\begin{array}{c}
\rho_{\mathrm{f}} \omega^{2} \mathbf{f}_{\mathrm{FEM}} \\
\mathbf{f}_{\mathrm{BEM}}
\end{array}\right)}_{\mathbf{f}},
$$

where the BEM matrix $\mathbf{B}$ was introduced to simplify the notation of equation (7). The matrix inverses in equation (8) are not evaluated explicitly, but conjugate gradient and GMRES iterations are applied on the FEM and BEM sub-systems, respectively. Instead of evaluating the exact matrix-vector product $\mathbf{S} \boldsymbol{\lambda}_{k}$ at each outer iteration step $k$, an approximation $\tilde{\mathbf{S}}_{k} \boldsymbol{\lambda}_{k}$ is computed. Using GMRES for the outer iterations, the precision of the inner approximation must be high for the first iterations on $\lambda$ and can be relaxed subsequently. An empirical relaxation strategy for a wide range of application is developed in [2]. For the solution of equation (8) with a target residual $\left\|\mathbf{f}-\mathbf{S} \boldsymbol{\lambda}_{k}\right\|_{2} \leq \epsilon^{\text {outer }}$, the inner systems must be solved with 


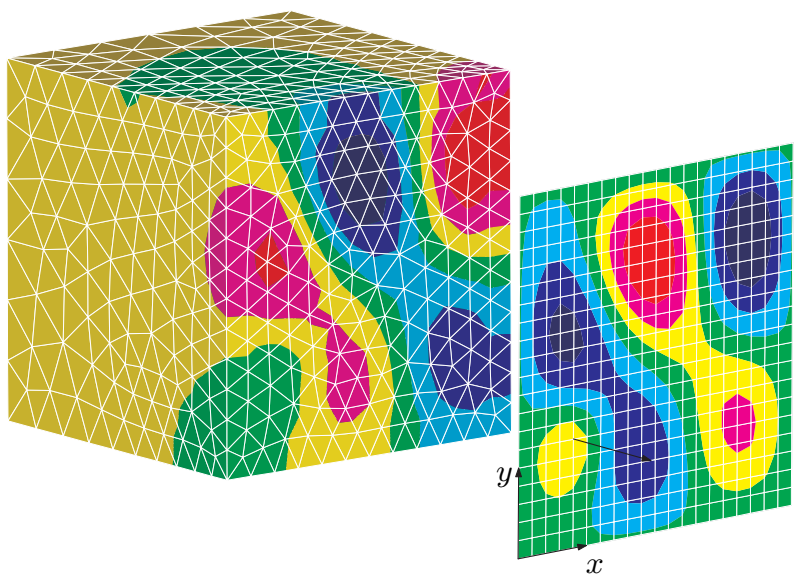

Figure 2: Surface pressure distribution and plate displacement at $180 \mathrm{~Hz}$.

a relative residual of

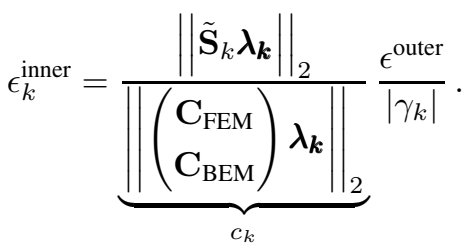

The value of the first ratio $c_{k}$ in equation (9) is not known prior to actually computing the approximations of the inner systems. Numerical experiments show that the value of the ratio usually does not change significantly from one outer iteration to the next. Thus, the ratio of the previous step can be used as a first guess to determine the required precision of the current approximation. Using the computed result, the true ratio is calculated and compared to the previous one. Only if there is a significant deviation, i.e. if the true ratio is significantly smaller than the guess, the approximation must be computed again up to the correct precision.

\section{Numerical example}

As a numerical example, the cavity backed elastic plate from [3] is chosen. Fig. 2 shows the surface pressure distrbution of the cavity and the plate displacement at $180 \mathrm{~Hz}$. The non-conforming discretization consists of 316 triangular boundary elements on the interface and $20 \times 20$ plate elements. 


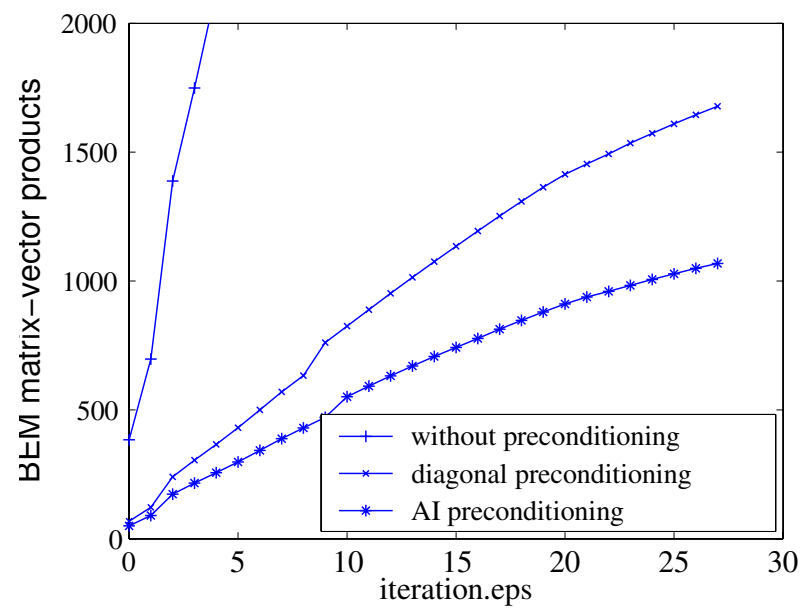

Figure 3: Preconditioning of BEM system for inexact Uzawa algorithm.

In Table 1 the numerical cost and the true residual are documented for the solution of the system arising from the example problem. The target residual of the outer GMRES is set to $10^{-4}$. Using the relaxation scheme, the numerical cost is reduced from 2018 BEM matrix-vector products to 1678 BEM matrix-vector products, i.e. the computing time is reduced by more than $15 \%$.

Table 1: Choice of residual $\epsilon_{k}^{\text {inner }}$ for solution of BEM system.

\begin{tabular}{ccccc}
\hline$\epsilon_{k}^{\text {inner }}$ & $\begin{array}{c}\text { outer } \\
\text { iterations }\end{array}$ & $\begin{array}{c}\text { BEM matrix- } \\
\text { vector products }\end{array}$ & GMRES resid. & true residual \\
\hline $\begin{array}{c}\text { fixed strategy } \\
c_{k} \epsilon^{\text {outer }}\end{array}$ & 28 & 2018 & $9.06 \cdot 10^{-5}$ & $1.03 \cdot 10^{-4}$ \\
\hline $\begin{array}{c}\text { relax. strategy } \\
c_{k} \epsilon^{\text {outer }} /\left|\gamma_{k}\right|\end{array}$ & 28 & 1678 & $9.06 \cdot 10^{-5}$ & $1.01 \cdot 10^{-4}$ \\
\hline
\end{tabular}

Preconditioning of the sub-systems is essential for the efficiency of the solver. As an advantage of the presented approach, standard preconditioners for the FEM and BEM parts can be used. The required number of matrix-vector products for approximating the inner BEM system without, with diagonal, and with approximate inverse preconditioning [4] is plotted in Fig. 3. The approximate inverse approach performs superior and is recommended for the solution of coupled BEM systems. 


\section{Conclusion}

For large scale models, standard BEM formulations are not applicable, since their numerical complexity scales as $N^{2}$. In the present paper a FMM algorithm was derived for the Helmholtz equation and adopted for the symmetric Galerkin BEM. Estimates of the numerical complexity for the FMM show that it allows to evaluate a matrix-vector product of the BEM with a computational cost of $\mathcal{O}\left(N \log ^{2} N\right)$ for simulations with a constant factor $\lambda / h$ which is typical for engineering applications. For mesh refinement at a constant frequency, the complexity is slightly higher, when increasing the expansion length to yield the convergence behavior of the BEM. For the solution of the system of equations GMRES with pre-conditioning by the single layer potential was employed, leading to iteration counts which are independent of the mesh size $h$. However, the number of iterations still depends considerably on the frequency, providing further research demands. The presented mortar coupling algorithm for structureacoustic field interaction allows the coupling of non-conforming discretizations. This can improve the efficiency of the coupled simulation since specialized ansatz functions and adapted element sizes can be used in the subdomains. As a further advantage, independent solvers and adapted preconditioning can be used for the approximation of the BEM and FEM subsystems in the proposed nested iteration scheme. In combination with the fast multipole boundary element method, a powerful simulation tool is available for acoustic-structure interaction. The discussed example shows that the method is suitable for applications where large-scale BEM models are required.

\section{References}

[1] Fischer, M., The Fast Multipole Boundary Element Method and its Application to Structure-Acoustic Field Interaction. PhD thesis, Institut A für Mechanik, Universität Stuttgart, 2004.

[2] Bouras, A. \& Frayssé, V., A relaxation strategy for inexact matrix-vector products for Krylov methods. CERFACS Technical Report TR/PA/00/15, 2000.

[3] Fischer, M. \& Gaul, L., Fast BEM-FEM mortar coupling for acoustic-structure interaction. International Journal for Numerical Methods in Engineering, 62, pp. 1677-1690, 2005.

[4] Fischer, M., Perfahl, H. \& Gaul, L., Approximate inverse preconditioning for the fast multipole BEM in acoustics. Computing and Visualization in Science, 8(3-4), pp. 169-177, 2005. 